\title{
Evolution of the atomic valence observed by the reaction fragility spectra on the reaction path
}

\author{
Jarosław Zaklika ${ }^{1} \cdot$ Ludwik Komorowski $^{1} \cdot$ Piotr Ordon $^{2}$ (D) \\ Received: 4 December 2018 / Accepted: 4 April 2019 / Published online: 26 April 2019 \\ (C) The Author(s) 2019
}

\begin{abstract}
The computational fragility spectra of atoms on the reaction path are presented for a selection of canonical processes represented by an amino group rotation around the $(\mathrm{X}) \mathrm{HC}-\mathrm{NH}(\mathrm{Y})$ bond $\left(\mathrm{X}=\mathrm{O}, \mathrm{S} ; \mathrm{Y}=\mathrm{H}, \mathrm{CH}_{3}\right)$. Calculated spectra are found to very accurately describe the variation of atomic valence. Significant linear correlation is also demonstrated between the Wiberg bond indices and the corresponding elements of the connectivity matrix, instrumental for calculation of the spectra. Demonstrated atomic fragility spectra contain rich and subtle information on the variation of the bonding status of all atoms, including the weak interacting individual hydrogens. Correlation with the atomic valences confirm the earlier finding that the spectra contain a picture of the electron density flow upon a reaction.
\end{abstract}

Keywords Reaction fragility spectrum $\cdot$ Hellmann-Feynman force $\cdot$ IRC reaction path $\cdot$ Reaction force $\cdot$ Reaction force constant

\section{Introduction}

We have studied molecular reactivity modifications along the course of chemical reaction for a long time [1-3]. We have developed regional chemical potential evolution and identified the most reactive state along the IRC reaction path [4]. Recently, we introduced a new concept of reaction fragility to identify reaction mechanism and to spot electron density modifications along the IRC reaction path $[5,6]$. We introduced a novel approach, aiming at observation of the electron density flow between atoms, rather than tracing the global parameters of the system. This requires focusing on atoms in the reacting

This paper belongs to Topical Collection 8th conference on Modeling \& Design of Molecular Materials (MDMM 2018)

Electronic supplementary material The online version of this article (https://doi.org/10.1007/s00894-019-4029-0) contains supplementary material, which is available to authorized users.

Piotr Ordon

piotr.ordon@upwr.edu.pl

1 Department of Physical and Quantum Chemistry, Wrocław University of Science and Technology, Wyb. Wyspiańskiego 27, 50-370 Wrocław, Poland

2 Department of Physics and Biophysics, Wrocław University of Environmental and Life Sciences, ul. Norwida 25, 50-373 Wrocław, Poland system. Instead of exploring the common population analyses, the Hellman-Feynman (H-F) forces on nuclei have been proposed for a description of an atom-in-molecule with no other arbitrary definition thereof [7].

The $\mathrm{H}-\mathrm{F}$ force on a nucleus is characterized by two features, essential from the chemical point of view. As demonstrated by Feynman [8], the force acting on a nucleus represents an effect of the electron density function in the entire system $\rho(\mathbf{r})$, and yet, it identifies the atom with no need to introduce some artificial partition of the electron density (Eq. 1).

$-\nabla_{A} E=\mathbf{F}_{A}+\mathbf{F}_{A}^{n-n}$

The H-F force $\left(\mathbf{F}_{A}\right)$ contains uniquely the effect of the whole electron density on a single nucleus:

$\mathbf{F}_{A}=\int \rho(\mathbf{r}) \varepsilon_{A}(\mathbf{r})$

The inter-nuclear interactions are separated in $\mathbf{F}_{A}^{n-n}$ :

$\mathbf{F}_{A}^{n-n}=\sum_{B \neq A}^{\text {atoms }} Z_{B} \varepsilon_{A}\left(\mathbf{R}_{B}\right)$

where

$\varepsilon_{A}(\mathbf{r})=-\nabla_{A} v(\mathbf{r})$

is the electric field due to the nucleus $A$ at point $\mathbf{r}$. 
Although the electron density function $\rho(\mathbf{r})$ in the integral (Eq. 2) spans over the whole system of nuclei, the $\mathbf{F}_{A}$ force value is dominated by the contribution from the bonds of the nucleus $A$ due to the effect of its strongest electric field (Eq. 4).

Variation of the electron density function $\rho(\mathbf{r})$ in a reaction progress must bring the corresponding variation of the $\mathrm{H}-\mathrm{F}$ force on atoms affected by this process.

An important feature of this analysis stems from the electrodynamical property of $\mathbf{F}_{A}^{n-n}$ :

$\nabla_{A} \cdot \mathbf{F}_{B}^{n-n}=0$

This is a direct consequence of the Laplace equation [9]. Calculating the divergence of the internuclear repulsion force, as specified by Yamaguchi et al. [10], leads to the same result. Thus, the second derivative of energy calculated from Eq. 1 as $-\nabla_{A}^{2} E$ contains the electronic term exclusively, the divergence of the $\mathrm{H}-\mathrm{F}$ force on an atom: $\nabla_{A} \cdot \mathbf{F}_{A}$. The divergences of force are combinations of elements of the common Hessian matrix; hence, this concept has opened new prospects for observation of the electron density modifications upon a reaction.

$a_{\xi}^{A} \equiv \frac{d}{d \xi}\left(\nabla_{A} \cdot \mathbf{F}_{A}\right)$

$a_{\xi}^{A}$ is the third derivative of energy; hence, it provides a measure of anharmonicity (cubic force constants) [11]. It describes the change of the force constant (the divergence) of the link of an atom (cumulative force constant) along the reaction path. The term fragility, used in other branches of chemistry, has been proposed for this quantity [6]. By the conceptual DFT analysis, an important albeit approximate conclusion has been arrived at: the electron density change around an atom in question is reflected by the atomic fragility $a_{\xi}^{A}$ (Eqs. 6 and 7).

$a_{\xi}^{A} \propto \frac{S}{N} \int \frac{d \rho(\mathbf{r})}{d \xi}\left[\varepsilon_{A}(\mathbf{r})\right]^{2} d \mathbf{r}$

Here $\mathrm{S}$ is the global softness. Significant contributions to the integral in Eq. 7 come from regions where the modification of the electron density upon a reaction step is nonnegligible $(d \rho(\mathbf{r}) / d \xi \neq 0)$ and the electric field of the nucleus is still sufficiently strong; $\left[\varepsilon_{A}(\mathbf{r})\right]^{2}$ value under the integral in Eq. 7 is recognized as the energy density of the electrostatic field generated by nucleus $A$. Thus, observation of the atomic fragility along the reaction path provides a spectrum of changes in the electron density in bonding regions of an atom in question [5].

Previously, computational studies of the molecular modifications upon intrinsic reaction coordinate have been brought closer to the chemical reality by the concept introduced by Toro-Labbé. The author proposed the reaction force be calculated as the energy derivative over the reaction progress $(\xi)$ for the system (super-molecule) traveling along the IRC path
[12-16]. The idea has been further developed by introducing the reaction force constant [17-19] (the second energy derivative over $\xi$ ) and another global parameter for the reaction, the electron electronic flux, an analogous derivative of the chemical potential $\mu$. Separation of these parameters into individual atoms has also been demonstrated [20,21]. However, the efforts to describe the actual flow of electrons between atoms remained inconclusive in this works [22].

This present work focused on the examination of the above conclusion by the routine calculation along the reaction path (IRC) of both the force divergences and the elements of the density matrix $\left(D_{i j}\right)$, explored in the Wiberg concept of the bond indices $\left(W_{A B}\right)$ and the atomic valence $\left(V_{A}\right)$ [23].

$W_{A B}=\sum_{i \in A} \sum_{j \in B}\left|D_{i j}\right|^{2}$ and $V_{A}=\sum_{B} W_{A B}$

For a test reaction, the well-recognized process of internal rotation was selected in formamide, thioformamide, acetamide, and $\mathrm{N}$-methyl-acetamide.

The hindered internal rotation around the $\mathrm{C}-\mathrm{N}$ bond in formamide and thioformamide has been intensively studied theoretically due to its importance for understanding the conformations of peptides and proteins [24, 25]. The rotational barrier in formamide is known to be lower than in its thioanalogue [26]. Laidig and Cameron have argued that the primary difference between the two molecules is greater donation of charge to the $\mathrm{NH}_{2}$ from the $\mathrm{CSH}$ group than from the $\mathrm{COH}$ group [27]. The ground state electronic delocalization was found to make the largest contribution to the torsional barrier by Mo et al. [28]. Ghanty \& Ghosh found, in their important DFT study, that $(\mathrm{S}) \mathrm{C}-\mathrm{N}$ bond order is higher that (O)C-N for the two analogues, and also the calculated change in the bond order in going from the planar to nonplanar structures is more for thioformamide [29].

\section{Methods}

The divergences of $\mathrm{H}-\mathrm{F}$ forces are available from the Cartesian Hessian as the appropriate sums of its elements (Eq. 9, ref. 5, 6).

$\nabla_{A} \cdot \mathbf{F}_{A}=\frac{\partial F_{A, x}}{\partial R_{A, x}}+\frac{\partial F_{A, y}}{\partial R_{A, y}}+\frac{\partial F_{A, z}}{\partial R_{A, z}} \equiv C_{A A}$

Summation of the atomic terms produces the trace of the Hessian, variable with the reaction progress (Eq. 10).

$\sum_{A} \nabla_{A} \cdot \mathbf{F}_{A}=\operatorname{Tr} \mathbf{C}$

The interatomic terms are calculated accordingly:

$\nabla_{B \neq A} \cdot \mathbf{F}_{A}=\frac{\partial F_{A, x}}{\partial R_{B, x}}+\frac{\partial F_{A, y}}{\partial R_{B, y}}+\frac{\partial F_{A, z}}{\partial R_{B, z}} \equiv C_{A B}$ 

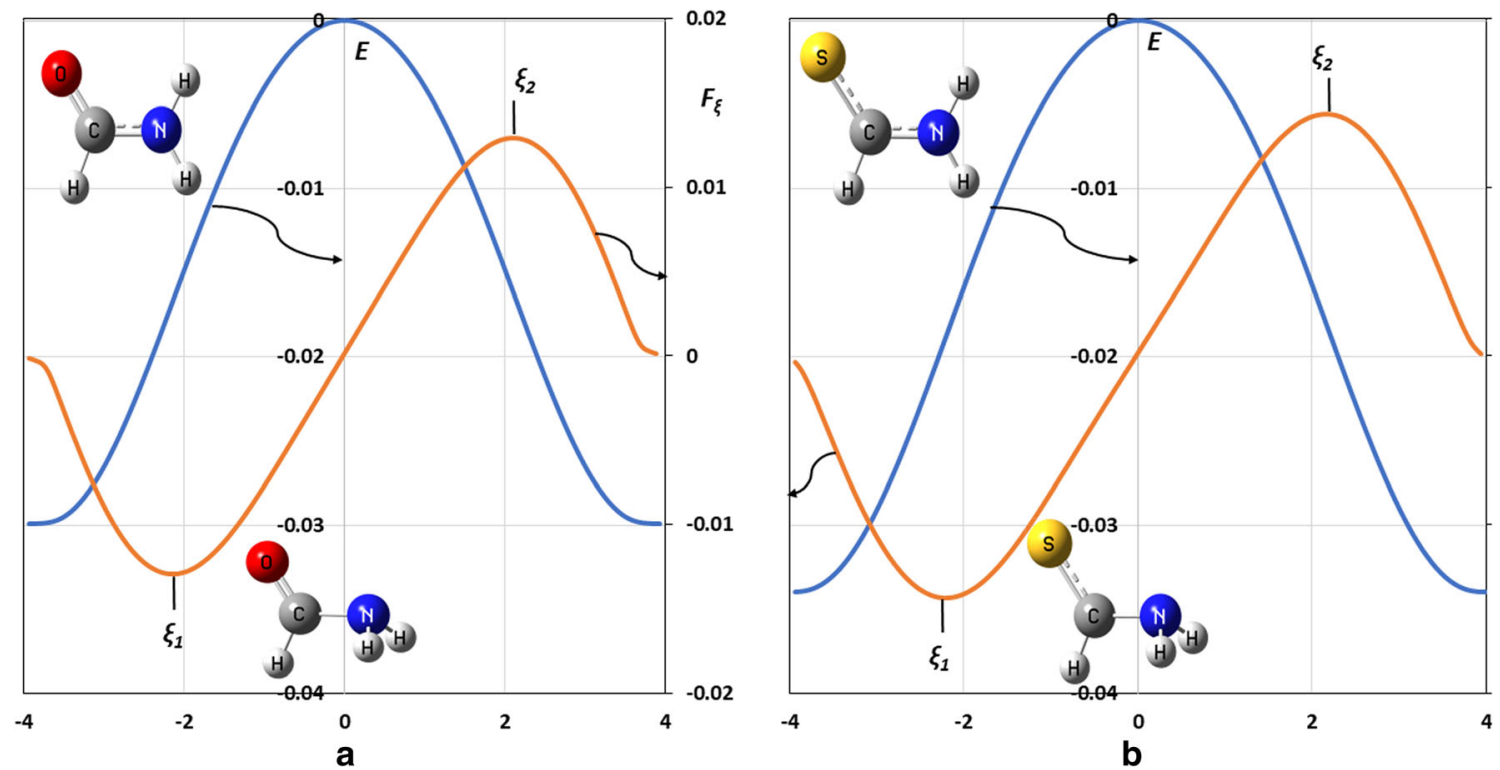

Fig. 1 Energy diagram normalized to the transition state value (in a.u.) and the reaction force span calculated for the formamide (a) and thioformamide (b) internal rotation over TS2 (see text). Position of the minimum and maximum reaction force are marked as $\xi_{1}$ and $\xi_{2}$ accordingly

By Eqs. 9 and 11 the entire connectivity matrix $\mathbf{C}(n \times n)$ of every system containing $n$ atoms is defined. Its diagonal elements are equal to the sum of corresponding non-diagonal ones in the column or the row of the matrix (cf. ref. 6):

$\nabla_{A} \cdot \mathbf{F}_{A}=-\sum_{B} \nabla_{B} \cdot \mathbf{F}_{A}$

The atomic fragilities (Eq. 6) are additive and allow for observation of the group fragilities $\left(a_{\xi}^{G}\right)$ as well as the reaction fragility $\left(a_{\xi}\right)$.

$a_{\xi}^{G} \equiv \sum_{A \in G} a_{\xi}^{A}=\sum_{A \in G} \frac{d}{d \xi}\left(\nabla_{A} \cdot \mathbf{F}_{A}\right)$

$a_{\xi} \equiv \frac{d}{d \xi}(\operatorname{Tr} \mathbf{C})$

The trace of the connectivity matrix $\mathbf{C}$ is identical to the trace of the Hessian matrix. Numerical results for the elements of the connectivity matrix were obtained from the IRC energy profile reproduced by the standard procedure [30] at the MP2 level using the 6-311++G $(3 d f, 3 p d)$ basis set and the Gaussian 09 code [31]. The reaction progress parameter $(\xi)$ was calculated in the mass-weighted coordinates with standard number ca. 150 points over every reaction path studied [32]. The Cartesian Hessian elements and the harmonic frequencies were calculated separately using the geometry of the structures resulting from the IRC. The Wiberg indices $W_{A B}$ for the formal bonds were calculated for each configuration of reactants on the reaction path; atomic valences $V_{A}$ were calculated according to Eq. 8. The effect of electronic flow was also assessed by the atomic charges calculated in three typical computational schemes Mulliken, Hirshfeld, and natural orbital population analysis (NPA).

Two transition state structures (TS) were identified for the internal rotation in formamide and thioformamide (for the rich literature on the subject cf. ref. 28). They differ by the position of the pyramidal $-\mathbf{N}^{*} \mathrm{H}_{2}$ group with respect to oxygen (or sulfur) in the COHN (CSHN) plane. TS1 has the nitrogen lone pair anti to the carbonyl oxygen and TS2 has the nitrogen lone pair syn to the carbonyl. For the purpose of this study the rotation over TS2 was considered in formamide and thioformamide, as it is characterized by higher energy, typically by $3 \mathrm{kcal} \mathrm{mol}^{-1}$ (ref. 25). For the pair acetamide/Nmethylacetamide, both TS1 and TS2 were considered.

\section{Results}

The energy diagrams for the internal rotation in two example molecules are shown in Fig. 1 ; the reaction force $\left(F_{\xi}=\right.$

Table 1 Transition state energies and the position of two extremes of the reaction force

\begin{tabular}{lllll}
\hline Molecule & Transition state & $\Delta \mathrm{E}_{\mathrm{TS}}\left[\mathrm{kcal} \mathrm{mol}^{-1}\right]$ & $\xi_{1}$ & $\xi_{2}$ \\
\hline Formamide & $\mathrm{TS} 2$ & 18.75 & -2.12 & 2.07 \\
Thioformamide & $\mathrm{TS} 2$ & 21.35 & -2.23 & 2.18 \\
Acetamide & $\mathrm{TS} 1$ & 15.05 & -2.04 & 1.98 \\
Acetamide & $\mathrm{TS} 2$ & 17.87 & -2.34 & 2.29 \\
N- methylacetamide & $\mathrm{TS} 1$ & 14.27 & -7.55 & 3.70 \\
N- methylacetamide & $\mathrm{TS} 2$ & 18.89 & -5.48 & 4.07 \\
\hline
\end{tabular}



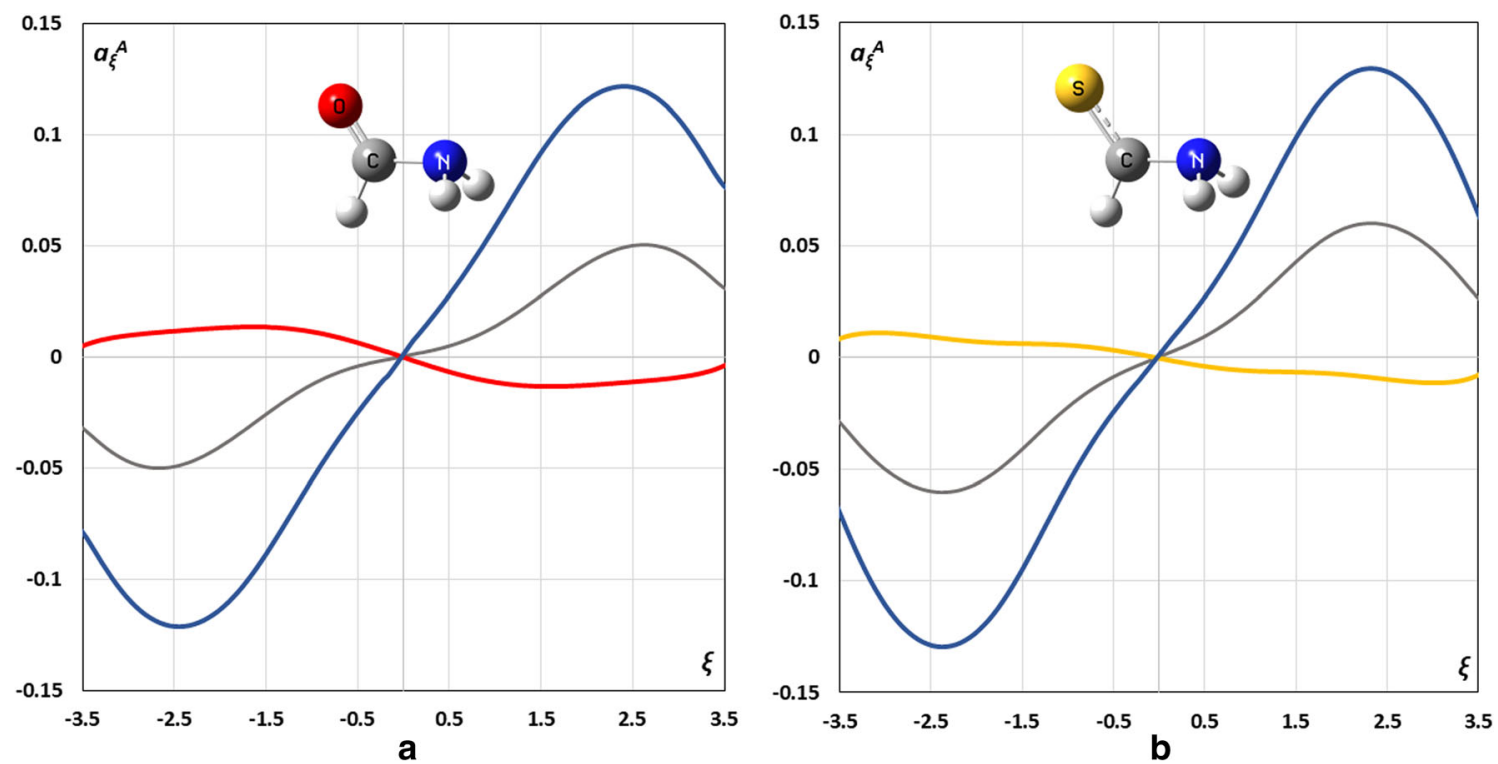

Fig. 2 The atomic fragilities $a_{\xi}^{A} \equiv \frac{d}{d \xi}\left(\nabla_{A} \cdot \mathbf{F}_{A}\right)$ for heavy atoms in formamide (a) and thioformamide (b) for the rotation over TS2. The lines correspond to atoms by their colors

$-d E / d \xi)$ is also shown. The reaction coordinates corresponding to the initial minimum $\left(\xi_{1}\right)$ and the final maximum $\xi_{2}$ of the reaction force are collected in Table 1 for all systems studied. The transition state energies reported in Table 1 are in agreement with the results by other authors [25]. The extremes of the reaction force reported in Table 1 serve as the routine guidelines indicating the reaction region supposedly dominated by the electron density changes, while the initial and final regions are viewed "as manifesting the identities of the reactant(s) and product(s), respectively" [33]. The atomic fragility spectra provide a critical test of this statement.

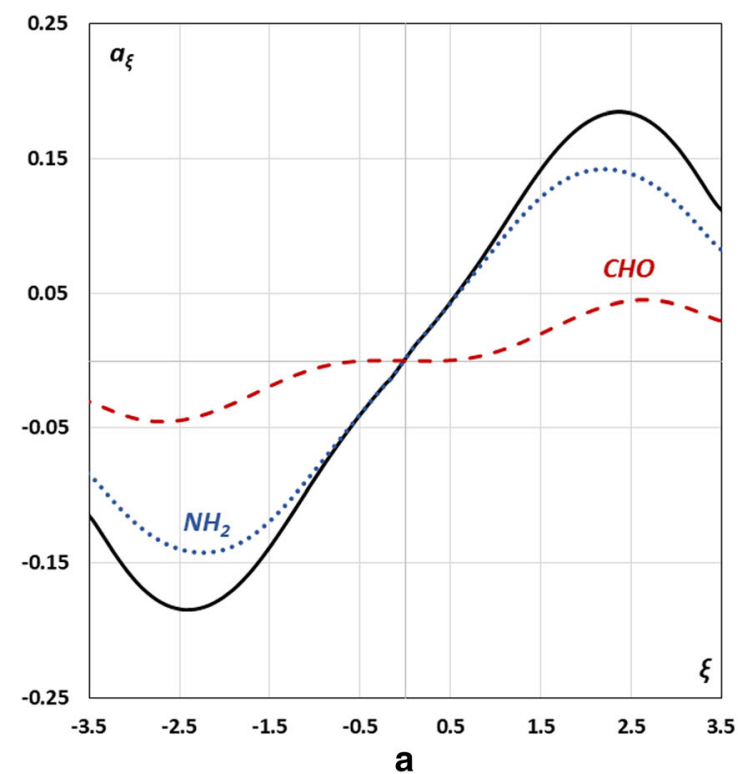

Fig. 3 The reaction fragility (Eq. 14, solid line) and the group fragilities (Eq. 13; $\mathrm{NH}_{2}$ and $\mathrm{CHO} / \mathrm{CHS}$ ) for the rotation in formamide (a) and thioformamide (b) over TS2 

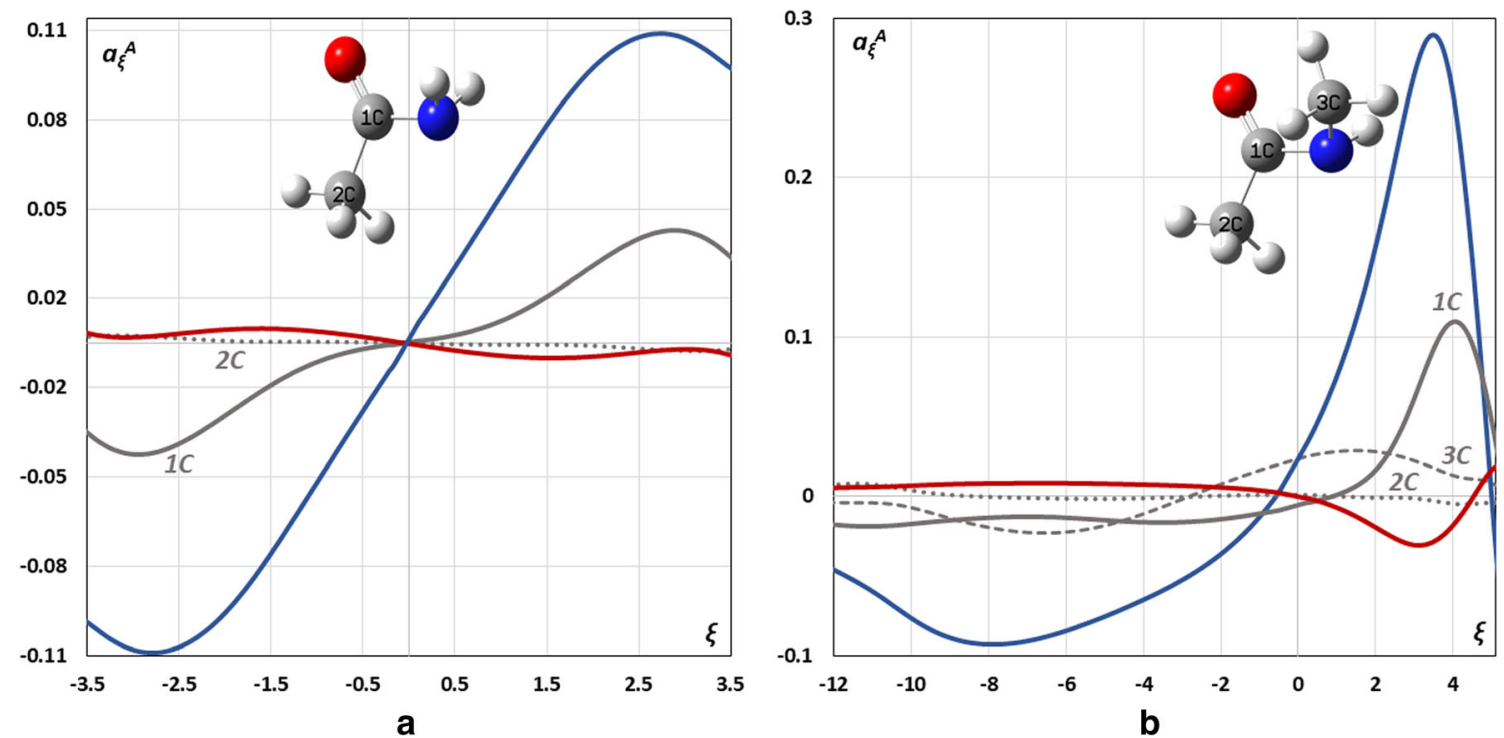

Fig. 4 The atomic fragilities (Eq. 5) for heavy atoms in the rotation over TS1 in acetamide (a) and N-methyl-acetamide (b). Lines correspond to atoms by colors, different carbon atoms are labeled

provide a minor contribution and are variable not only due to the electron transfer along the peptide bond but also as a consequence of the rotation of the group they belong to as a whole $\left(\mathrm{CH}_{3}\right)$. Effects of the subtle movements of an example hydrogen atom (NH in N-methylacetamide) are shown separately.

Effects of the subtle movements of an example hydrogen atom (NH in N-methylacetamide) are shown separately. The atomic fragility changes (Eq. 6) for heavy atoms in formamide and thioformamide molecules are shown in Fig. 2, the group fragilities for the rotation in these two molecules are in Fig. 3. The results for acetamide/N-methylacetamide pairs are shown in Figs. 4 and 5 (for TS1) and Figs. 6 and 7 (for TS2). The

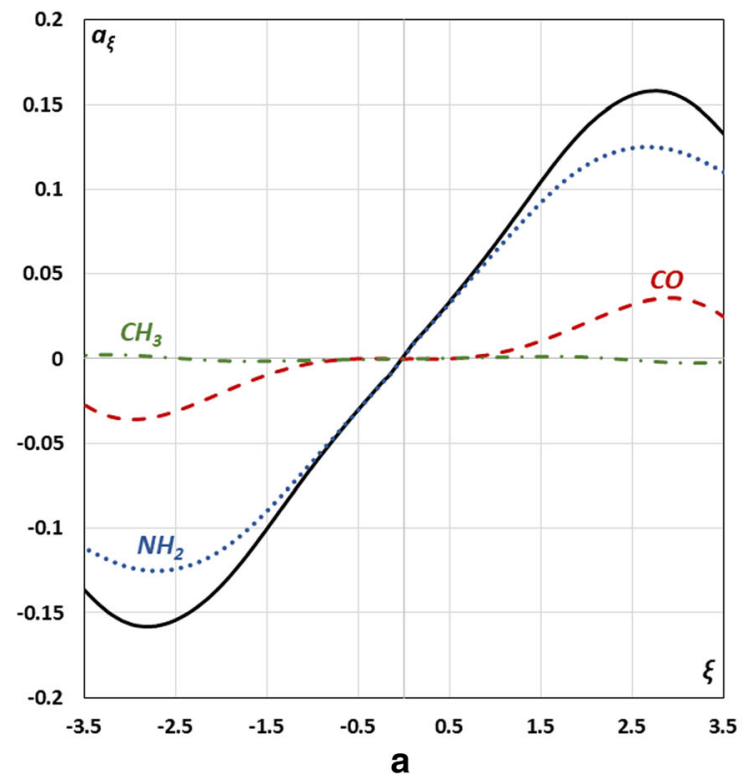

diagrams of atomic fragilities ("spectra") for the formamide and thioformamide (Fig. 2) very clearly show two downward peaks for $\mathrm{C}$ and $\mathrm{N}$ atoms describing the synchronous density decay in the region between these two atoms at the initial stage of the reaction. The process starts as early as at $\xi=-3.5$ and reaches its extreme at ca $\xi=-2.5$; the change is reversed symmetrically in the final reaction region. This observation indicates that the essential electron flow occurs far from the TS for these reactions (cf. Eq. 7), in the initial and final regions.

The characteristic difference between the oxygen atom in formamide and the sulfur atom in thioformamide is well demonstrated in their fragility spectra. Both tend to gradually gain

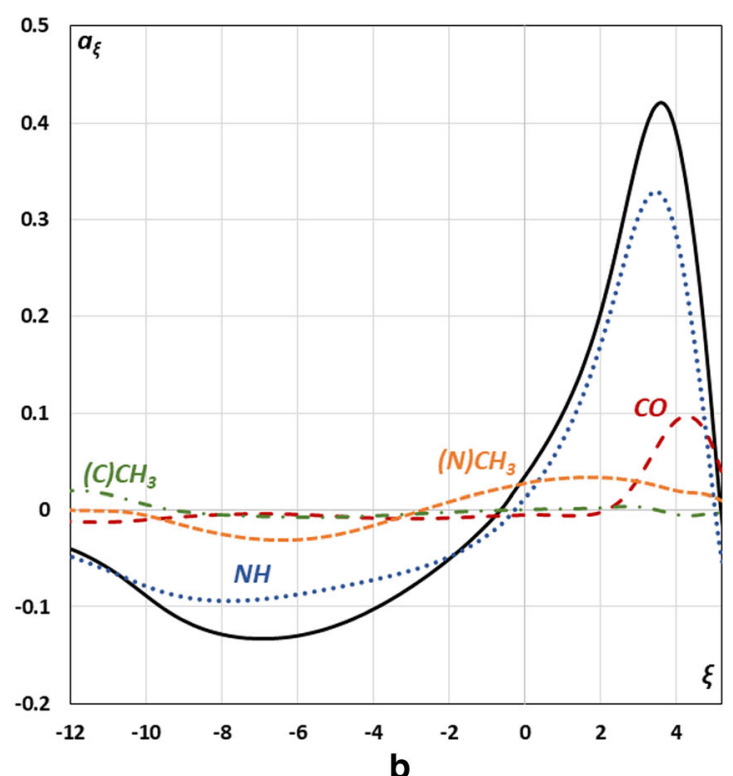

Fig. 5 The reaction fragility (Eq. 14, solid line) and the group fragilities (Eq. 13: $\mathrm{NH}_{2} \& \mathrm{NH}, \mathrm{CO}, \mathrm{CH}_{3}$ ) for the rotation over TS1 in acetamide (a) and $\mathrm{N}$ methyl-acetamide (b) 

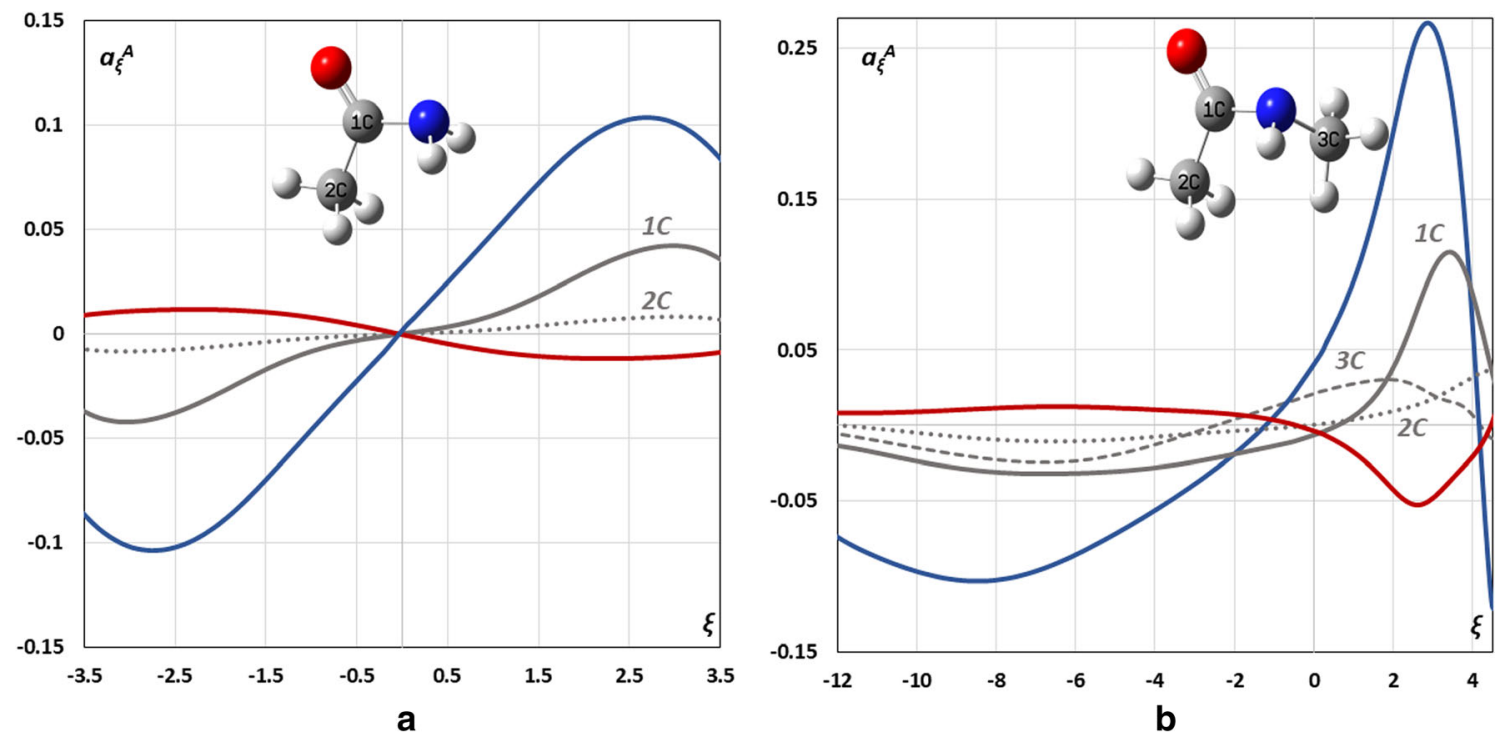

Fig. 6 The atomic fragilities (Eq. 5) for heavy atoms in the rotation over TS2 in acetamide (a) and N-methyl-acetamide (b). Lines correspond to atoms by colors, different carbon atoms are labeled

the density in their bonds upon the rotation; the corresponding flat upward peaks appear at $\xi=-1.4$ and $\xi=-3.4$, respectively. The much earlier response of sulfur atom to the rotation may result from its significantly larger softness, as compared to oxygen atom. The group fragility spectra in Fig. 3 provide yet more information: by the density decrease in the $\mathrm{C}-\mathrm{N}$ bond, the $\mathrm{NH}_{2}$ group is considerably more affected than the $\mathrm{CHO}$ group (or CHS). This matches the common chemistry expectation: the decrease of $\mathrm{C}-\mathrm{N}$ bond order is compensated for the carbon atom by the increase in $\mathrm{C}-\mathrm{O}$ bond order (confirmed by the $W_{\mathrm{CO}}$ index), while the excess charge on the nitrogen atom has no where to go. The reaction fragility extremes are larger in thioformamide than in formamide, which is well in accord with the higher TS energy in the thio-analogue. The extremes appear roughly at points where the reaction force also has its peaks (Fig. 1): apparently, $F_{\xi}$ correctly describes the general trend in this reaction, while atomic fragilities provide more detailed information on the density flow between individual atoms.

The spectra of acetamide are slightly more complex, although their general shape is similar. The notable effect is the character of the methyl group (Figs. 4a and 5a). When the rotation goes over TS1, neither the group nor its central carbon is affected by the process. For the rotation over TS2, both the carbon (Fig. 6a) and the methyl group as a whole (Fig. 7a) loses its bonding with the rest of the molecule. The
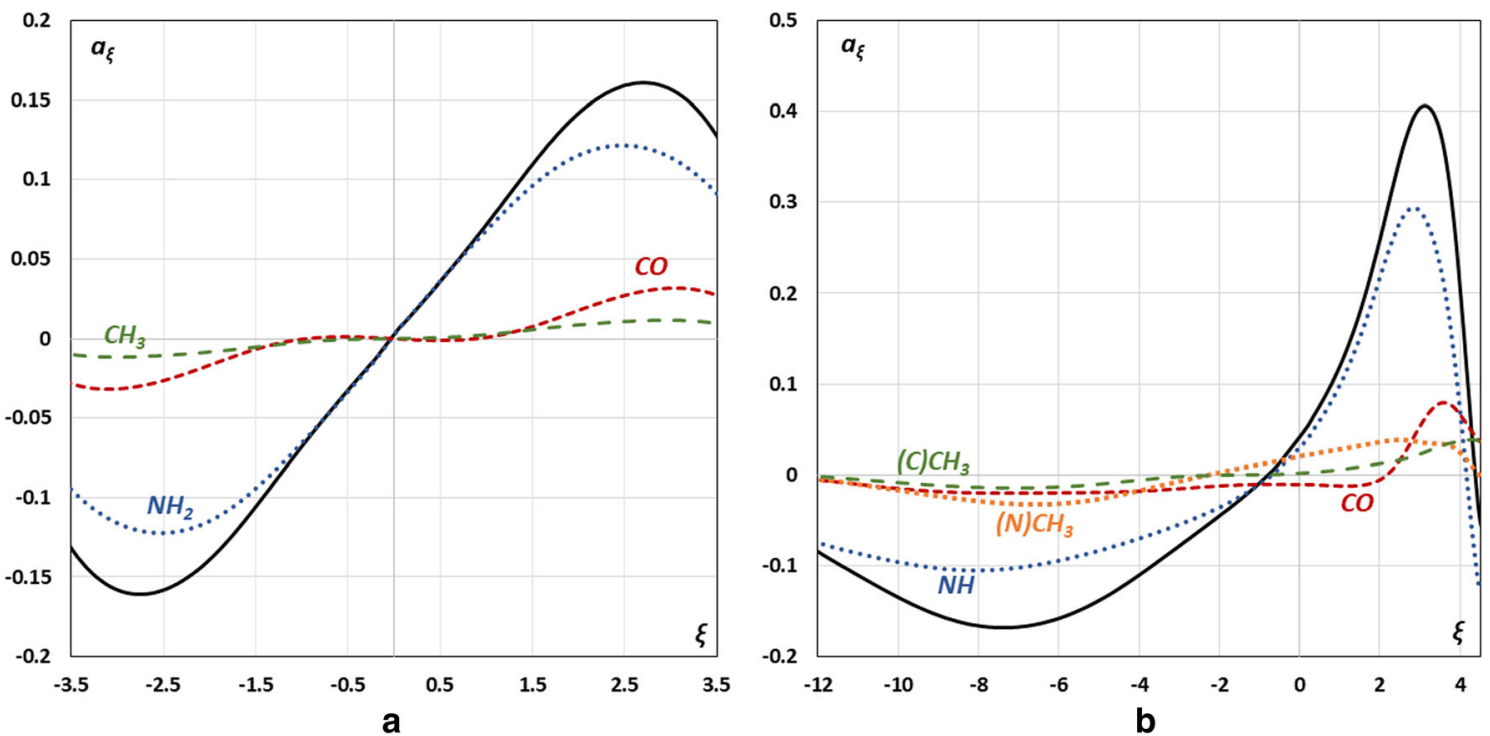

Fig. 7 The reaction fragility (Eq. 14, solid line) and the group fragilities (Eq. 13: $\mathrm{NH}_{2} \& \mathrm{NH}, \mathrm{CO}, \mathrm{CH}_{3}$ ) for the rotation over TS2 in acetamide (a) and Nmethyl-acetamide (b) 
Fig. 8 Hydrogen atom fragility for the $(\mathrm{N}) \mathrm{H}$ atom in $\mathrm{N}$-methylacetamide molecules rotating around the $\mathrm{C}-\mathrm{N}$ bond over TS1 and TS2, respectively

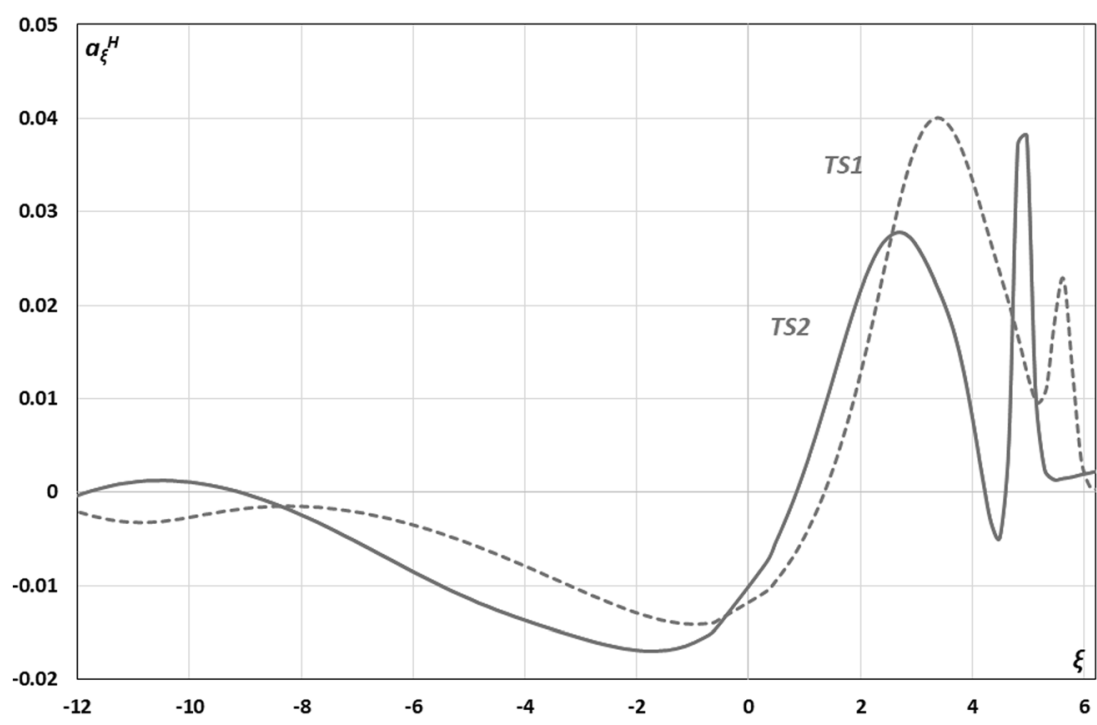

explanation is suggested by inspection of the structures TS1 and TS2 (Figs. 4a and 6a): rotation over TS2 (but not over TS1) is brought about through space interactions between $\mathrm{NH}_{2}$ hydrogens and $\mathrm{CH}_{3}$ hydrogens. This effect is even more evident in the $\mathrm{N}$-methyl-acetamide spectra.

Rotation in N-methyl-acetamide is considered as the twostep process starting from a molecule with the cis configuration of the two methyl groups with respect to the $\mathrm{C}-\mathrm{N}$ bond. Step 1 is $180^{\circ}$ rotation to TS1, ending at the trans configuration. Step 2 is further rotation by $180^{\circ}$ over TS 2 back to the initial trans rotamer. The principal process again lowers the bond order between $\mathrm{N}$ and $1 \mathrm{C}$ (Figs. 4b and 6b); however, the role of carbon at the initial stage is less clear that it was in acetamide. Only the group fragilities expose the effect (Figs. 5b and 7b): the CO group as a whole loses its contact with neighbors very slowly but regains it abruptly in the final stage. The interactions between methyl groups $\left(\mathrm{N}-\mathrm{CH}_{3}\right.$ and $\mathrm{C}-\mathrm{CH}_{3}$ ) are clearly lost at the beginning of step 1 and are regained at the end of step 2. Otherwise, the $\mathrm{C}-\mathrm{CH}_{3}$ methyl group is nonactive, in contrast to $\mathrm{N}-\mathrm{CH}_{3}$ clearly responding to the rotation especially at step 1 (Figs. $4 \mathrm{~b}$ and $5 \mathrm{~b}$ ).

\section{Observation of the hydrogen movement}

The atomic fragility of the single $(\mathrm{N}) \mathrm{H}$ hydrogen is chosen as an example (Fig. 8), leading to important observation of a more general nature. Fragilities of hydrogen are ca. one order of magnitude smaller that they are for atoms participating in the electron density flow vital for the reaction. Nevertheless, precision of the numerical data for the connectivity matrix diagonal elements in routine calculations (Eq. 9) is sufficient to reproduce the fine details of the derivative defined by Eq. 6 (hydrogen fragility $a_{\xi}^{H}$ ). The origin of the remarkable peaks in the final stage of the rotation may be assessed from visualization of this process (see files in the attached Electronic Supplementary Material). They appear to be a consequences of the interactions $(\mathrm{N}) \mathrm{H}$ with the neighboring atoms, rather than an effect of the electron density flow along the $\mathrm{N}-\mathrm{C}-\mathrm{O}$ skeleton.

By inspection of the animations it may be supposed that the broad minima in the initial phase are associated with the gradual transformation of the $\mathrm{sp}^{2}$ to $\mathrm{sp}^{3}$ hybridization of the nitrogen atom over the rotation by $120^{\circ}(\xi<0)$, the $\mathrm{N}-\mathrm{H}$ bond order is lowered. The final part of the rotation (by $60^{\circ}, \xi>0$ ) demonstrates two mysterious effects: regaining the bond order typical for the $\mathrm{sp}^{2}$ hybrid (first peak) followed by lowering it when the $(\mathrm{N}) \mathrm{CH}_{3}$ group does the same (the downward peak) and finally completing the process.

Table 2 Linear correlation parameters for the relation between the nondiagonal elements of the connectivity matrix (Eq. 11) calculated for the bonds between atoms in the $\mathrm{O}-\mathrm{C}-\mathrm{N}$ skeleton of the molecules and the Wiberg indices of corresponding bonds (Eq. 8): $W_{X Y}=A \cdot\left[-C_{X Y}\right]+B$

\begin{tabular}{llrrl}
\hline Molecule & Bond & \multicolumn{1}{l}{ A } & \multicolumn{1}{l}{$\mathrm{B}$} & $\mathrm{R}^{2}$ \\
\hline Formamide & $\mathrm{C}-\mathrm{O}$ & 5.31 & -3.08 & 0.988 \\
$-3.9<\xi<3.9$ & $\mathrm{~N}-\mathrm{C}$ & 1.25 & 0.50 & 0.980 \\
Thioformamide & $\mathrm{C}-\mathrm{S}$ & 11.30 & -3.38 & 0.986 \\
$-3.9<\xi<4.0$ & $\mathrm{~N}-\mathrm{C}$ & 1.37 & 0.49 & 0.991 \\
Acetamide TS1 & $\mathrm{C}-\mathrm{O}$ & 4.16 & -1.96 & 0.984 \\
$-4.2<\xi<4.2$ & $\mathrm{~N}-\mathrm{C}$ & 1.13 & 0.57 & 0.994 \\
Acetamide TS2 & $\mathrm{C}-\mathrm{O}$ & 4.24 & -2.04 & 0.998 \\
$-4.2<\xi<4.2$ & $\mathrm{~N}-\mathrm{C}$ & 1.25 & 0.57 & 0.981 \\
N-methyl-acetamide TS1 & $\mathrm{C}-\mathrm{O}$ & 3.38 & -1.23 & 0.967 \\
$-13.6<\xi<8.9$ & $\mathrm{~N}-\mathrm{C}(\mathrm{O})$ & 1.20 & 0.53 & 0.998 \\
N-methyl-acetamide TS2 & $\mathrm{C}-\mathrm{O}$ & 3.71 & -1.52 & 0.951 \\
$-14.8<\xi<7.2$ & $\mathrm{~N}-\mathrm{C}(\mathrm{O})$ & 1.34 & 0.47 & 0.974 \\
\hline
\end{tabular}


Table 3 Linear correlation parameters for the relation between the diagonal elements of the connectivity matrix (Eq. 11) calculated for atoms in the $\mathrm{O}-\mathrm{C}-\mathrm{N}$ skeleton of the molecules and the Wiberg valency (Eq. 8): $V_{X}=A \cdot C_{X X}+B$

\begin{tabular}{lllrl}
\hline Molecule & Atom & \multicolumn{1}{l}{ A } & \multicolumn{1}{l}{ B } & \multicolumn{1}{l}{$\mathrm{R}^{2}$} \\
\hline Formamide & $\mathbf{O}$ & 3.17 & -1.08 & 0.997 \\
$-3.9<\xi<3.9$ & $\mathbf{C}$ & 0.083 & 3.62 & 0.978 \\
& $\mathbf{N}$ & 0.68 & 1.84 & 0.990 \\
Thioformamide & $\mathbf{S}$ & 7.61 & -1.69 & 0.994 \\
$-3.9<\xi<4.0$ & $\mathbf{C}$ & -0.30 & 4.32 & 0.991 \\
& $\mathbf{N}$ & 0.92 & 1.54 & 0.988 \\
Acetamide TS1 & $\mathbf{O}$ & 6.12 & -3.82 & 0.969 \\
$-4.2<\xi<4.2$ & $(\mathrm{O}) \mathbf{C}$ & 0.21 & 3.42 & 0.980 \\
& $\mathbf{N}$ & 0.70 & 1.81 & 0.991 \\
Acetamide TS2 & $\mathbf{O}$ & 3.06 & -0.98 & 0.997 \\
$-4.2<\xi<4.2$ & $(\mathrm{O}) \mathbf{C}$ & 0.057 & 3.70 & 0.925 \\
& $\mathbf{N}$ & 0.64 & 1.89 & 0.992 \\
N-methyl-acetamide TS1 & $\mathbf{O}$ & 3.84 & -1.65 & 0.959 \\
$-13.6<\xi<8.9$ & $(\mathrm{O}) \mathbf{C}$ & 0.23 & 3.38 & 0.925 \\
& $\mathbf{N}$ & 0.78 & 1.88 & 0.973 \\
N-methyl-acetamide TS2 & $\mathbf{O}$ & 3.13 & -1.01 & 0.935 \\
$-14.8<\xi<7.2$ & $(\mathrm{O}) \mathbf{C}$ & 0.17 & 3.50 & 0.976 \\
& $\mathbf{N}$ & 0.73 & 1.96 & 0.955 \\
& & & &
\end{tabular}

\section{Bond orders and connectivity matrix}

The bond orders calculated by the Wiberg formula (Eq. 8) are compared to the negative of the corresponding non-diagonal elements of the connectivity matrix (Eq. 11). The general linear correlation is found for the atoms other that the hydrogen over the entire span of reaction coordinate (Table 2 ) for the $\mathrm{C}-$ $\mathrm{O}$ and $\mathrm{N}-\mathrm{C}$ bonds that are principal targets of this observation in the entire set of the molecules studied. For bonds to external groups $\left(\mathrm{CH}_{3}\right)$, the correlation is limited to the regions only. This is a consequence of the slight rotational motion of methyl upon the reaction that affects the $\mathrm{C}-\mathrm{C}$ and $\mathrm{C}-\mathrm{N}$ bonds outside the $\mathrm{O}-\mathrm{C}-\mathrm{N}$ skeleton in acetamide and $\mathrm{N}$ - methylacetamide, as is clearly observed in the $\mathrm{CH}_{3}$ group fragility in Figs. 5 and 7. The $\mathrm{R}^{2}$ parameters indicated in Table 2 confirm the meaningful character of the linear correlations.

\section{Atomic valences and the diagonal elements of the connectivity matrix}

Linear correlations are also observed between the diagonal elements of the connectivity matrix and the atomic valences as defined by Wiberg (Eq. 8). The results are presented in Table 3 for $\mathrm{O}, \mathrm{C}$, and $\mathrm{N}$ atoms participating in the electron density flow in the common structural skeleton of the molecules. Parameter B of the linear correlation shows weak dependence on the chemical environment, while the slope (A) substantially varies between atoms.

\section{Discussion and conclusions}

The most important finding of this work is perhaps the discovered linear relation between the non-diagonal elements of the connectivity matrix and the Wiberg bond indices (Table 1). The correlation is clear for atoms directly participating in the electron flow. For the side atoms (e.g., rotating methyl groups), correlation is affected by other variable interactions, sometimes even between distant atoms. The origin of the two quantities is quite different: Wiberg indices are derived from the parameters of the wave function (cf. ref. 23), while the connectivity matrix elements are energy derivatives. The Wiberg indices are widely accepted as a practical measure of the electron density modifications; the effort was once made by Lendvay to observe their evolution with the reaction progress [34]. The discovered simple relation of the indices to the elements of the connectivity matrix tends to corroborate the original suggestion that the fragility spectra indeed reproduce the electron density change between atoms.

The correlations are even more important when the relation between the valency of atoms and the corresponding diagonal elements of the connectivity matrix is considered (Table 2). Here, the correlation parameters must be related to the properties of one atom only. They seem to be characteristic for the type of an atom: observed average slopes of the correlation for $\mathrm{N}, \mathrm{O}$, and $\mathrm{C}$ atoms are $0.64( \pm 0.1), 3.3( \pm 0.3)$, and $0.1( \pm 0.2)$; for a single example of sulfur it is 7.61. The suggested a priori relation (Eq. 7) may be helpful in finding the general solution of this problem.

The atomic fragility spectra provide important information for a chemist, more vital than observation of a chosen bond: how much and in what direction the valency of an atom is modified upon a reaction. Given the precision of the data and relative simplicity of their extraction, the atomic fragility spectra provide an attractive tool for data compression that is greatly desired in chemistry (advocated by Mayer in his study, ref. 23), leading to a comprehensible yet quantitative picture of the electron density evolution in a chemical reaction.

Acknowledgments The work was financed by a statutory activity subsidy from the Polish Ministry of Science and Higher Education for the Faculty of Chemistry of Wrocław University of Science and Technology ref. No 0401/0121/18. The use of resources of Wrocław Center for Networking and Supercomputing is gratefully acknowledged (WCSS grant No 249 and GW 036).

Open Access This article is distributed under the terms of the Creative Commons Attribution 4.0 International License (http:// creativecommons.org/licenses/by/4.0/), which permits unrestricted use, distribution, and reproduction in any medium, provided you give appropriate credit to the original author(s) and the source, provide a link to the Creative Commons license, and indicate if changes were made. 


\section{References}

1. Ordon P, Tachibana A (2007) J Chem Phys 126:234115

2. Ordon P, Tachibana A (2005) J Chem Sci 117:583

3. Ordon P, Tachibana A (2005) J Mol Model 11:312

4. Jędrzejewski M, Ordon P, Komorowski L (2013) J Mol Model 19: 4203-4207

5. Ordon P, Komorowski L, Jędrzejewski M (2017) J Chem Phys 147: 134109

6. Komorowski L, Ordon P, Jẹdrzejewski M (2016) Phys Chem Chem Phys 18:32658

7. Jędrzejewski M, Ordon P, Komorowski L (2016) J Phys Chem A 120:3780-3787

8. Feynman RP (1939) Phys Rev 56:340

9. Jackson JD (1975) Classical electrodynamics, 2nd edn. Wiley, New York

10. Yamaguchi Y, Osamura Y, Goddard JD, Schaefer IIIHF (1994) A new dimension to quantum chemistry: analytic derivative methods in ab initio molecular electronic structure theory. Oxford University Press, New York

11. Komorowski L, Ordon P (2004) Int J Quantum Chem 99:153-160

12. Toro-Labbé A (1999) J Phys Chem A 103(22):4398-4403

13. Politzer P, Toro-Labbé A, Gutierrez-Oliva S, Herrera B, Jaque P, Concha MC, Murray JS (2005) J Chem Sci (India) 117(5):467-472

14. Toro-Labbe A, Gutierrez-Oliva S, Murray JS, Politzer P (2007) Mol Phys 105(19-22):2619-2625

15. Politzer P, Toro-Labbé A, Gutiérrez-Oliva S, Murray JS (2012) Advances in Quantum Chemistry 64:189-210 [Sabin JR, Brändas EJ eds] Elsevier, Amsterdam

16. Toro-Labbé A, Gutiérrez-Oliva S, Politzer P, Murray JS (2009) In: Chattaraj PK (ed) Chemical reactivity theory. A density functional viewpoint. CRC, Boca Raton, pp 293-302

17. Jaque P, Toro-Labbé A, Politzer P, Geerlings P (2008) Chem Phys Lett 456(4-6):135-140

18. Yepes D, Donoso-Tauda O, Perez P, Murray JS, Politzer P, Jaque P (2013) Phys Chem Chem Phys 15:7311-7320

19. Yepes D, Murray JS, Politzer P, Jaque P (2012) Phys Chem Chem Phys 14:11125-11134

20. Vöhringer-Martínez E, Toro-Labbé A (2012) J Phys Chem A 116: $7419-7423$
21. Inostroza-Rivera R, Yahia-Ouhmed M, Tognetti V, Joubert L, Herrera B, Toro-Labbé A (2015) Phys Chem Chem Phys 17: 17797-17807

22. Martínez JI, Toro-Labbé A (2015) J. Phys Chem C 119:3040-3049

23. Mayer I (2007) J Comput Chem 28:204-221

24. Galvez J, Lopez S, Jose I, Guirado A (2015) Comput Theor Chem 1069:40-47

25. Kang YK, Park HS (2004) J Mol Struct THEOCHEM 676(1-3): 171-176

26. Wiberg KB, Rablen PR (1995) J Am Chem Soc 117(8):2201-2209

27. Laidig KE, Cameron LM (1996) J Am Chem Soc 118(7):17371742

28. Mo Y, Schleyer PR, Wu W, Lin M, Zhang Q, Gao J (2003) J Phys Chem A 107(46):10011-10018

29. Ghanty TK, Ghosh SK (2000) J Phys Chem A 104(13):2975-2979

30. Miller WH, Handy NC, Adams JE (1980) J Chem Phys 72:99

31. Frisch MJ, Trucks GW, Schlegel HB, Scuseria GE, Robb MA, Cheeseman JR, Scalmani G, Barone V, Mennucci B, Petersson GA, Nakatsuji H, Caricato M, Li X, Hratchian HP, Izmaylov AF, Bloino J, Zheng G, Sonnenberg JL, Hada M, Ehara M, Toyota K, Fukuda R, Hasegawa J, Ishida M, Nakajima T, Honda Y, Kitao O, Nakai H, Vreven T, Montgomery Jr JA, Peralta JE, Ogliaro F, Bearpark M, Heyd JJ, Brothers E, Kudin KN, Staroverov VN, Kobayashi R, Normand J, Raghavachari K, Rendell A, Burant JC, Iyengar SS, Tomasi J, Cossi M, Rega N, Millam JM, Klene M, Knox JE, Cros JB, Bakken V, Adamo C, Jaramillo J, Gomperts R, Stratmann RE, Yazyev O, Austin AJ, Cammi R, Pomelli C, Ochterski JW, Martin RL, Morokuma K, Zakrzewski VG, Voth GA, Salvador P, Dannenberg JJ, Dapprich S, Daniels AD, Farkas Ö, Foresman JB, Ortiz JV, Cioslowski J, Fox DJ (2009) Gaussian 09, revision A.02. Gaussian Inc., Wallingford

32. Piela L (2007) Ideas of quantum chemistry. Section 14.3: intrinsic reaction coordinate (IRC) or statics. Elsevier, Amsterdam, pp 781-3

33. Yepes D, Murray JS, Santos JC, Torro-Labbe A, Politzer P, Jaque P (2013) J Mol Model 19:2689-2697

34. Lendway G (1994) J Phys Chem 98:6098-6104

Publisher's note Springer Nature remains neutral with regard to jurisdictional claims in published maps and institutional affiliations. 